\title{
Systematic review of mental health disorders and intimate partner violence victimisation among military populations
}

\author{
Katherine Sparrow $^{1} \cdot$ Jamie Kwan $^{2} \cdot$ Louise Howard $^{3} \cdot$ Nicola Fear $^{4}$. \\ Deirdre MacManus ${ }^{1}$
}

Received: 11 March 2017/ Accepted: 14 July 2017/Published online: 26 July 2017

(c) The Author(s) 2017. This article is an open access publication

\begin{abstract}
Purpose There is growing awareness of the problem of intimate partner violence (IPV) among military populations. IPV victimisation has been shown to be associated with mental disorder. A better understanding of the link between IPV and mental disorder is needed to inform service development to meet the needs of military families. We aimed to systematically review the literature on the association between IPV victimisation and mental health disorders among military personnel.

Methods Searches of four electronic databases (Embase, Medline, PsycINFO, and Web of Science) were supplemented by reference list screening. Heterogeneity among studies precluded a meta-analysis.

Results Thirteen studies were included. There was stronger evidence for an association between IPV and depression/ alcohol problems than between IPV and PTSD. An
\end{abstract}

Electronic supplementary material The online version of this article (doi:10.1007/s00127-017-1423-8) contains supplementary material, which is available to authorized users.

Deirdre MacManus

deirdre.macmanus@kcl.ac.uk

1 Forensic and Neurodevelopmental Sciences Department, Institute of Psychiatry Psychology and Neuroscience, King's College London, PO23, 16 De Crespigny Park,

London SE5 8AF, UK

2 Department of Psychological Medicine, Weston Education Centre, 10 Cutcombe Road, London SE5 9RJ, UK

3 David Goldberg Centre, Institute of Psychiatry Psychology and Neuroscience, King's College London, 16 De Crespigny Park, London SE5 8AF, UK

4 King's Centre for Military Health Research, King's College London, Weston Education Centre, 10 Cutcombe Road, London SE5 9RJ, UK association between IPV and mental health problems was more frequently found among veterans compared to active duty personnel. However, the link between IPV and alcohol misuse was more consistently found among active duty samples. Finally, among active duty personnel psychological IPV was more consistently associated with depression/ alcohol problems than physical/sexual IPV. The review highlighted the lack of research on male IPV victimisation in the military.

Conclusions There is evidence that the burden of mental health need may be significant among military personnel who are victims of IPV. The influence of attitudes towards gender in the military on research in this area is discussed. Further research is needed to inform development of services and policy to reduce IPV victimisation and the mental health consequences among military personnel.

Keywords Military $\cdot$ Mental disorder $\cdot$ Intimate partner violence $\cdot$ Review

\section{Introduction}

Intimate partner violence (IPV) is a serious, preventable public health problem that occurs in all settings and among all religious, cultural, and socioeconomic groups [1]. IPV includes physical and sexual violence, stalking, and psychological aggression (including coercive controlling behaviour) by a current or former intimate partner [2]. It has been estimated to account for up to $7 \%$ of the overall burden of disease among women, primarily due to mental health impairment [3, 4]. IPV research among military populations lags behind that in civilian populations [5-7]. The well-publicised murders of the wives of four 
American Fort Bragg soldiers in the space of 6 weeks in 2002 [8] led to increased awareness within international Armed Forces communities of the reality of IPV among military couples. Military couples ${ }^{1}$ are exposed to some unique stressors which have been shown to impact negatively on relationships and some of which have been associated with increased risk of IPV [9], including operational deployments and deployment related injuries [10-13], combat exposure [13, 14] and service-related mental health and behavioural problems $[15,16]$, frequent relocation, and familial separation [17-19]. The experience of military service and the consequences of some of these stressors can continue to impact on relationships long after the serving person has left the Armed Forces [20-29]. Transitioning out of the military is also associated with a range of additional psychosocial stressors [30-35] and veterans have been shown to report high levels of some mental health problems [36-41]. It should, therefore, not be assumed that the correlates of IPV are consistent across civilian and military couples [42, 43] and they may even differ between military couples with an active serving partner and those with a partner who is a veteran [5, 41].

There is a growing body of research on IPV victimisation among military populations, though these studies are very heterogeneous in terms of samples, method of measurement of IPV, and definitions of different types of IPV. To our knowledge, no UK studies exist. Studies in the US have found high levels of IPV victimisation among military personnel, both male and female [44-49], with conflicting conclusions on whether IPV victimisation is higher among males or females, depending on the severity of violence measured $[50,51]$. Many of the other risk factors for IPV victimisation in the general population have been found to be important among military populations also such as age [52-55], social class [56], and level of education [53, 57], though findings are not consistent [44, 52, 56-59]. It has been suggested by some studies that IPV may be more prevalent among military than civilian populations $[46,60,61]$, though this has also not been a universal finding [62].

There is a large body of literature which has established the link between IPV victimisation and mental disorder in the general population. Research has focused on depression, PTSD, anxiety, eating disorders, substance misuse, and chronic mental illness more broadly, with the most consistent evidence highlighting a link between IPV and depression, followed by PTSD and anxiety disorders [63-72]. There is evidence to suggest a causal association between IPV and mental disorders in both directions: IPV can lead to negative mental health outcomes, and mental health problems can render a person more vulnerable to

\footnotetext{
${ }^{1}$ We use 'military couple' to mean a couple in which one or both the partners is serving or has served in the military.
}

experiencing IPV [73]. A recent systematic review found evidence for an association between IPV perpetration and mental disorders among military populations [74]. A number of studies have also explored the association between IPV victimisation and mental disorders among military personnel. The methodological rigour and hence the findings of these studies have varied greatly. A systematic review of such studies is needed to gain a better understanding of the link between IPV victimisation and mental health problems, such as depression, PTSD, anxiety disorders, and substance misuse, among military personnel, to inform the development of services to meet the needs of military families.

The aim of this study was, therefore, to systematically review extant studies to summarise the literature exploring IPV victimisation and specific mental health problems among male and female military personnel (both serving and ex-serving).

\section{Methods}

A literature search was undertaken for studies examining mental health problems associated with IPV victimisation among military populations. Searches of the following electronic databases were carried out: Embase, Medline, PsycINFO, and Web of Science. The search terms and combinations used were identical for all four databases. Search results were limited to papers published in English. In addition to searching bibliographic databases, the reference lists of all relevant papers and reviews were searched. Authors were contacted to request raw data where necessary. This review followed PRISMA reporting guidelines and the protocol is registered with PROSPERO: registration CRD42016044119.

Studies were eligible for inclusion if they: (1) involved male and/or female serving or ex-serving military personnel; (2) reported the risk of IPV victimisation among those with and without mental disorder or vice versa, and/or a measure of association between IPV and mental disorder; (3) measured IPV using a validated tool or adapted question(s); (4) measured mental health using a validated diagnostic or screening tool, e.g., the PTSD checklist (PCL), or the Alcohol Use Disorders Identification Test (AUDIT); (5) presented the results of peer reviewed research based on any quantitative study design capable of providing the data listed above; and (6) had a sample size of over 100 participants. IPV was defined as "any incident of threatening behaviour, violence or abuse (psychological, physical, sexual, financial, or emotional) between adults who are or have been intimate partners regardless of gender or sexuality" [75]. Mental disorders included schizophrenia and psychotic disorders, mood disorders, neurotic and 
stress-related disorders (including anxiety disorders and post-traumatic stress disorder), eating disorders, and mental and behavioural disorders related to alcohol or substance misuse. Titles and abstracts were screened against the inclusion criteria. The full texts of potentially eligible studies were then reviewed. Quality appraisal of the included studies was conducted independently by two reviewers using a checklist adapted from validated tools [76-80] (see supplementary information). Agreement for the overall quality appraisal scores for the 13 studies was calculated using the Kappa statistic (Kappa=0.74). A third, more senior, reviewer was consulted in the instance of any scoring discrepancies. Studies that scored $50 \%$ or higher on criteria relating to selection bias were categorised as high quality. This review focused on studies which allowed the estimation of risk of IPV among individuals with and without mental health disorder. The $50 \%$ criterion was selected to identify studies with a lower risk of selection bias and on whose findings greater weight could be placed. Qualitative and quantitative data were extracted from included studies, including information on study design, sample characteristics, and measurement tools used, as well as data on the risk of IPV victimisation and mental disorder. Data were extracted separately for men and women, where possible.

Figure 1 describes the study selection process. Literature searches yielded 6809 unique references; 6745 were excluded following title and abstract screening and a further 51 were excluded following full-text screening. The remaining 13 papers were included in this review. All 13 papers were identified through searches of electronic databases. References identified through other sources (i.e., screening the reference lists of included studies) were all duplicates. The 13 papers reported on a combined sample of 55,883 participants.

Heterogeneity among the studies in this review (primarily regarding the timing and type of IPV studied) precluded a meta-analysis.

\section{Results}

\section{Key features of included studies}

The key characteristics of the included studies are summarised in Table 1. All studies were conducted in highincome countries, with two conducted in Canada [44, 45], and the other 11 conducted in the USA. Five of the 13 studies were conducted in clinical settings [52, 81-84] and eight in non-clinical settings [44, 45, 47, 48, 50, 54, $58,85]$. Five of the 13 studies were categorised as high quality (i.e., scoring $50 \%$ or higher for selection bias) $[44,45,47,48,50]$.
As shown in Table 1, nine studies reported on female victims, three studies reported on male victims, and two studies included both males and females, but did not report mental health outcomes by gender. Seven studies explored the link between IPV and depression, five studies focused on IPV and PTSD, and five on IPV and alcohol problems (see Table 2). Six of the 13 studies utilised validated measures of IPV such as the Conflict Tactics Scale (CTS) or the Abuse Assessment Screen to measure domestic violence. Four studies used adapted versions of validated tools [54, 58, 81, 83]. The remaining three studies measured IPV using an objective question [47, 48, 52] (see Table 3).

Studies examined IPV measured over a variety of time periods. Four studies reported on past-year IPV [50, 54, 84, 85], six studies on lifetime [47, 48, 58, 81-83], two studies on IPV experienced over the course of the current relationship [44, 45], and one study on IPV experienced during military service [52]. Further details of sample size, study methods, and findings are presented in Tables 2 and 3 .

\section{Main findings}

\section{Depression}

Nine studies examined depressive symptoms among individuals who have experienced IPV victimisation [44, 45, 47, 48, 52, 54, 58, 84, 85], with four studies rated as high quality [44, 45, 47, 48]. Six studies found a statistically significant association between IPV victimisation and depression after taking account of potential confounders [44, 45, 47, 48, 54, 84], of which four were rated as high quality.

The majority of study findings will be reported according to gender. However, two high-quality studies explored the association between depression and IPV experienced over the course of the current relationship among samples of male and female Canadian Armed Forces (CAF) members and did not stratify analyses by gender. The first study used a representative sample of 1745 CAF members and found that 'probable depression' was significantly associated with increased emotional and/or financial abuse, but not with any physical and/or sexual IPV [44]. The second study ( $n=529$ ) similarly found that emotional violence victimisation (defined as experiencing threats of violence) was significantly associated with depression [45], but physical violence victimisation was not.

Female victims In a study of past-year IPV among active duty females married to civilian spouses $(n=248)$, the researchers grouped participants according to six different patterns of violence (depending on gender of perpetrator 


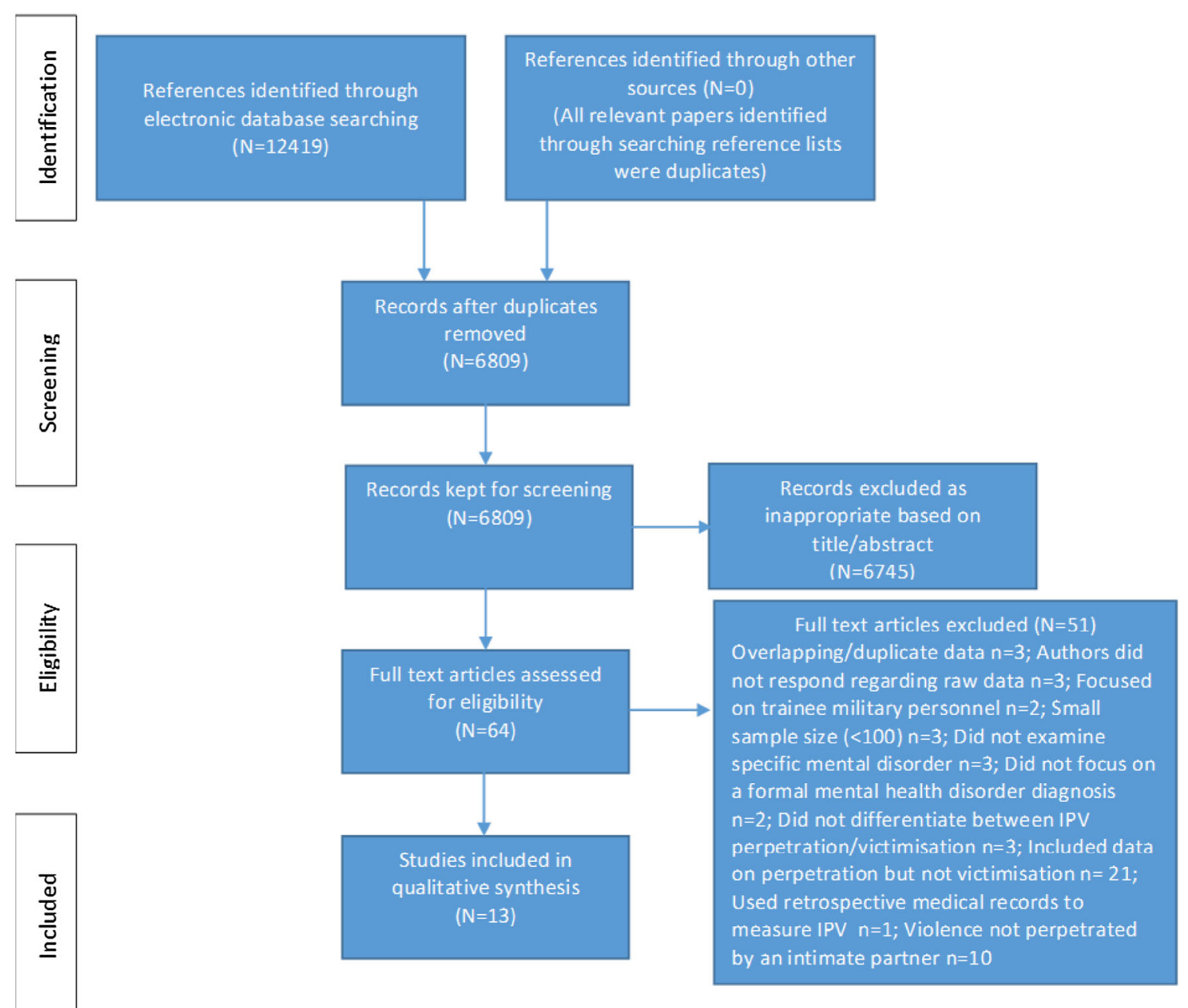

Fig. 1 Flow of information through the phases of the systematic literature search

and unidirectional/bi-directional violence of differing severities). It was found that mean depression scores were significantly higher among females who reported experiencing violence by a male civilian spouse compared to those who reported no violence. Mean depression scores did not differ significantly between the no violence group and the group in which the more severe violence was perpetrated by the enlisted female. The mean depression score for the group in which both the enlisted female and her spouse had engaged in severe violence and/or injury was significantly higher than all other group scores [85]. This study did not conduct a statistical analysis of the association between depression and IPV. Another study which utilised a clinical sample of female Veterans Affairs (VA) patients found that of those who reported any type of IPV (defined as physical and/or psychological) victimisation in the past year $67.2 \%$ were categorised as cases of depression, compared to $18.6 \%$ of those who did not report IPV. On further analysis, any past-year IPV was significantly associated with depression [84].
With regard to lifetime IPV, a high-quality study utilised data from a nation-wide telephone survey of non-institutionalised adults in the US and found that among female veterans (503 out of a total $n=21,162$ females) IPV was significantly associated with increased cases of depression [47] (see also Table 3). By comparison, a study of active duty females $(n=616)$ found no significant association between physical and/or sexual IPV and cases of depression [58]. Finally, a study exploring sexual IPV experienced by female VA patients $(n=369)$ during military service found no significant difference between the mean depression score of women who experienced sexual IPV compared to those who had not experienced sexual abuse [52].

Male victims One study of male active duty Army personnel $(n=488)$ found that past-year physical and psychological aggression was significantly associated with depression [54]. The researchers split their sample according to ethnicity and found that severe physical IPV victimisation was more strongly associated with depression 
Table 1 Key features of included studies

\begin{tabular}{|c|c|}
\hline & Total $(n=13)$ \\
\hline \multicolumn{2}{|l|}{ Study design } \\
\hline Cross-sectional & 13 \\
\hline \multicolumn{2}{|l|}{ Gender $^{\mathrm{a}}$} \\
\hline No. of papers reporting on male victims & $3[6,8,9]$ \\
\hline $\begin{array}{l}\text { No. of papers reporting on female } \\
\text { victims }\end{array}$ & $9[7,8,33-39]$ \\
\hline $\begin{array}{l}\text { Papers reporting on male and female } \\
\text { victims together-unable to get } \\
\text { separate data }\end{array}$ & $2[10,11]$ \\
\hline \multicolumn{2}{|l|}{ Setting } \\
\hline Clinical setting & $5[33-36,39]$ \\
\hline General military setting & $6[8-11,38,39]$ \\
\hline Community & $2[6,7]$ \\
\hline \multicolumn{2}{|l|}{ Sample } \\
\hline Air force (serving) & $1[8]$ \\
\hline Army (serving) & $2[8,9]$ \\
\hline Veterans & $7[6,7,33-37]$ \\
\hline Armed forces (all services-serving) & $3[10,11,39]$ \\
\hline \multicolumn{2}{|l|}{ Timing of IPV } \\
\hline Past-year & $4[8,9,36,38]$ \\
\hline Lifetime & $6[6,7,33-35,39]$ \\
\hline Over course of current relationship & $2[10,11]$ \\
\hline During military service & $1[37]$ \\
\hline \multicolumn{2}{|l|}{ Type of IPV } \\
\hline Physical & $5[9,10,33-35]$ \\
\hline Sexual & $2[34,37]$ \\
\hline Psychological/emotional & $5[6,8-10,34]$ \\
\hline Any IPV—(varying definitions) & $6[6,7,11,36,38,39]$ \\
\hline \multicolumn{2}{|l|}{ IPV measure } \\
\hline Validated tool & $6[8,10,11,34,36,38]$ \\
\hline Modified version of validated tool & $4[9,33,35,39]$ \\
\hline Objective IPV question & $3[6,7,37]$ \\
\hline \multicolumn{2}{|l|}{ Quality appraisal score } \\
\hline Low quality & $4[33-36]$ \\
\hline Medium quality & $4[9,37-39]$ \\
\hline High quality & $5[6-8,10,11]$ \\
\hline
\end{tabular}

${ }^{a}$ As categories (Gender and Type of IPV) are not mutually exclusive, totals may exceed 13

for Black than for White soldiers. Similarly, a high-quality community-based study (4356 male veterans out of a total $n=13,765$ males) found that among veterans lifetime IPV (any IPV defined as actual or threatened physical violence or unwanted sex) was significantly associated with increased depression [48].

\section{Post-traumatic stress disorder (PTSD)}

Five studies analysed the association between IPV victimisation and PTSD [44, 52, 58, 83, 84], with one study rated as high quality [44]. Three studies (none were high quality) found a significant association between IPV and PTSD after taking account of potential confounders [52, 83, 84]. Again, study results will mostly be reported by gender, but one high-quality study of CAF members which utilised a mixed gender sample did not stratify analyses by gender. This study found no significant association between any physical and/or sexual IPV or any emotional and/or financial IPV experienced over the course of the current relationship and PTSD [44].

Female victims A study of 160 female VA patients found that $41.4 \%$ of females who reported past-year IPV had a PTSD diagnosis, compared to $19.6 \%$ of those who did not report IPV, and past-year physical and/or psychological IPV was found to be significantly associated with PTSD [84]. Similarly, a study of female VA patients $(n=1206)$ found that $32.2 \%$ of those who reported lifetime IPV had a PTSD diagnosis, compared to $14.8 \%$ of those who did not report IPV, and PTSD was significantly associated with physical IPV [83]. In contrast, a military population-based study of active duty tri-service females $(n=616)$ did not find a significant association between physical and/or sexual lifetime IPV and PTSD [58]. Finally, one study found that women who experienced sexual IPV during military service had significantly higher levels of PTSD symptoms compared to women without a history of sexual IPV [52]. The PTSD scores of women who experienced sexual IPV by an intimate partner were not significantly different to those of women who experienced sexual abuse perpetrated by a non-intimate partner.

\section{Alcohol/substance use problems}

Seven studies explored alcohol misuse among individuals who have experienced IPV [44, 50, 54, 81, 82, 84, 85], with two studies rated as high quality [44, 50]. Four studies found a statistically significant association after taking account of potential confounders [44, 50, 54, 82] with two of these being high quality. One high-quality study with a mixed gender active duty sample did not stratify analyses by gender. It was found that experience of any emotional and/or financial abuse victimisation over the course of the current relationship was associated with high-risk drinking [44]. No significant association was found between physical and/or sexual IPV and high-risk drinking.

Female victims A high-quality study utilising a representative sample of active duty US Air Force members ( $n=42,744 ; 8031$ females) found that past-year clinically significant emotional abuse (defined as at least one reported act that caused significant distress that interfered with the victim's functioning) was significantly associated with 


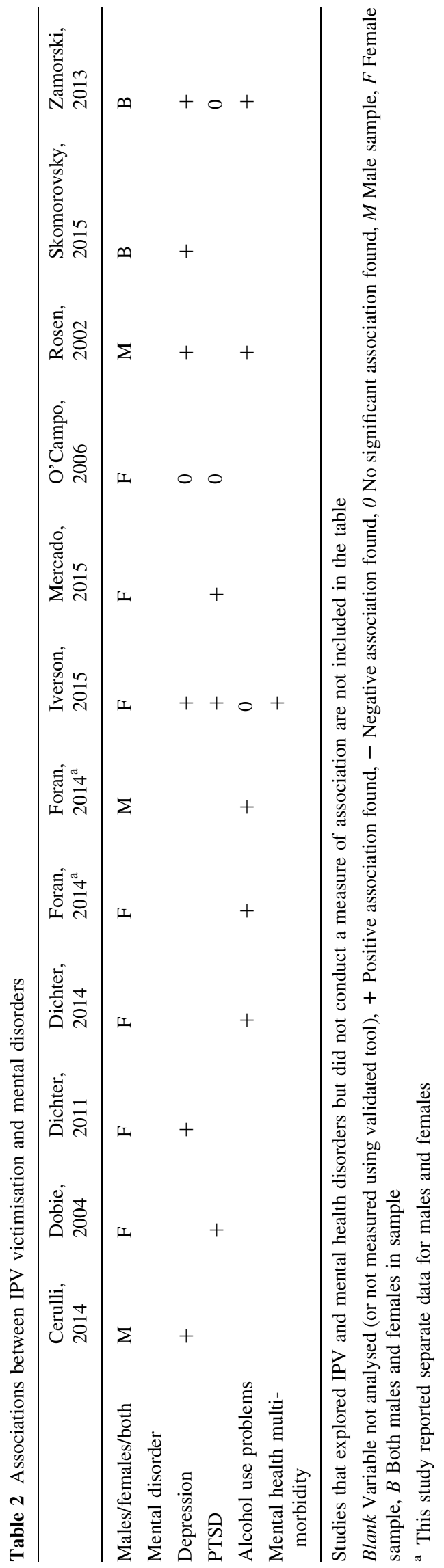

alcohol problems [50]. In contrast, two smaller and lower quality studies did not find different drinking patterns among those reporting past-year IPV compared to those without IPV [85] and did not find an association between IPV and probable alcohol dependence [84].

Two studies examined lifetime IPV victimisation and alcohol problems among clinical populations of female VA patients [81, 82]. One study $(n=249)$ found a significant association between sexual IPV (with/without physical or psychological IPV) and problem drinking, but no association with either physical (with/without psychological IPV) or psychological IPV [82]. The other study $(n=2670)$ reported that lifetime physical IPV increased significantly with AUDIT-C scores of five or more [81]. However, no statistical analysis of the association between IPV and alcohol problems was conducted (see also Table 3 ).

Male victims A high-quality study of 34,713 male US Air Force members found that clinically significant emotional abuse was significantly associated with alcohol problems [50]. Another study of active duty males found that severe physical past-year IPV and psychological IPV were associated with alcohol problems [54]. No significant association was found between mild physical IPV and alcohol problems.

\section{Mental health multi-morbidity}

A study of 160 female VA patients found that of those who reported IPV, 50\% reported mental health multi-morbidity (defined as the presence of at least two of the following conditions: depression, PTSD, alcohol dependence), compared to $20.6 \%$ of those who did not report IPV. Past-year physical and/or psychological IPV victimisation was significantly associated with mental health multi-morbidity [84].

\section{Discussion}

\section{Summary of main findings}

The aim of this review was to explore the association between IPV victimisation and mental health problems among current and former military personnel. The number and quality of studies which found an association between IPV and depression/alcohol problems was higher than for IPV and PTSD. An association between IPV and mental health problems was more frequently found in studies of veterans compared to active duty personnel. However, the link between IPV and alcohol misuse was more consistently found among active duty samples. Among active duty personnel, psychological IPV was more consistently 


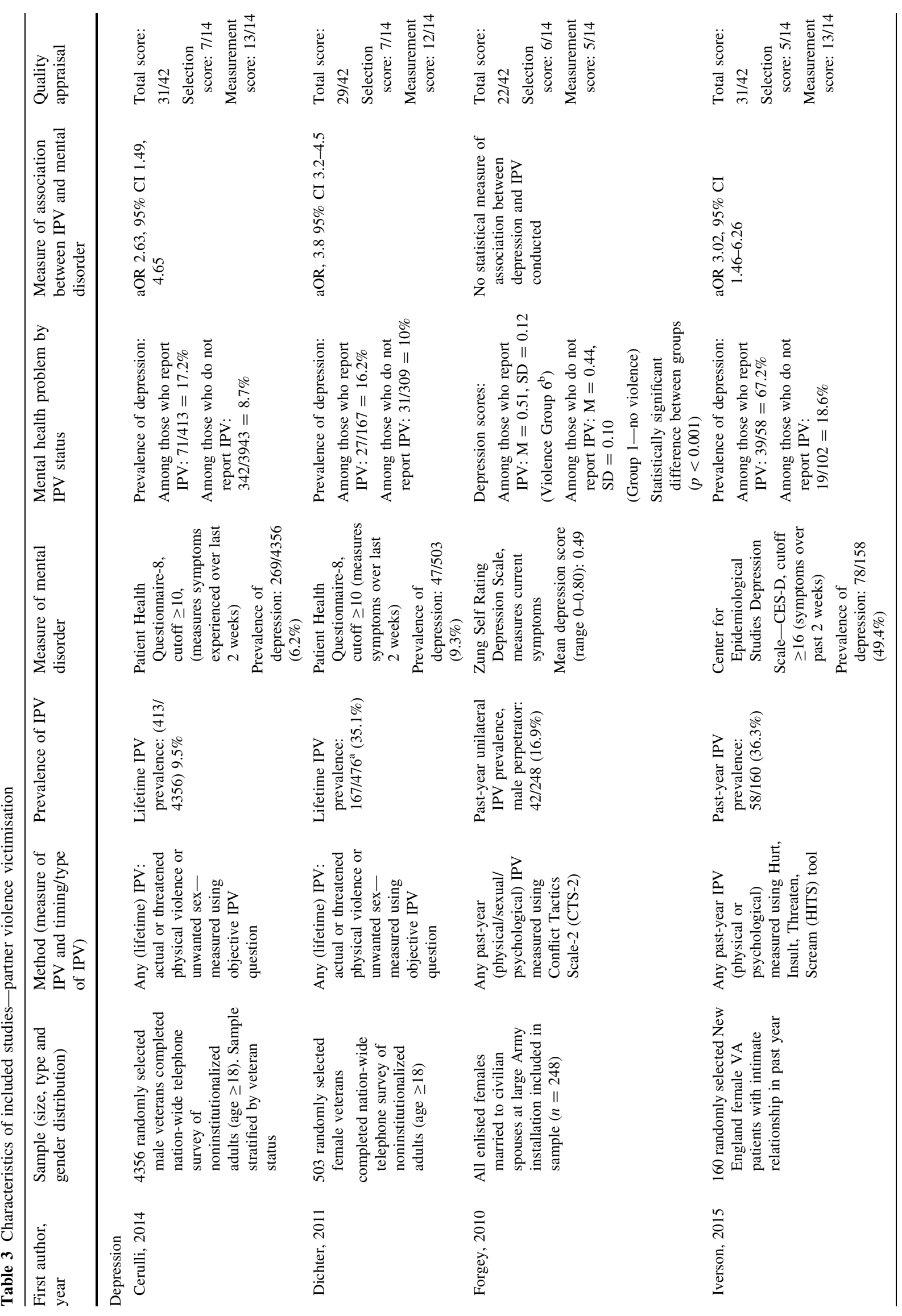




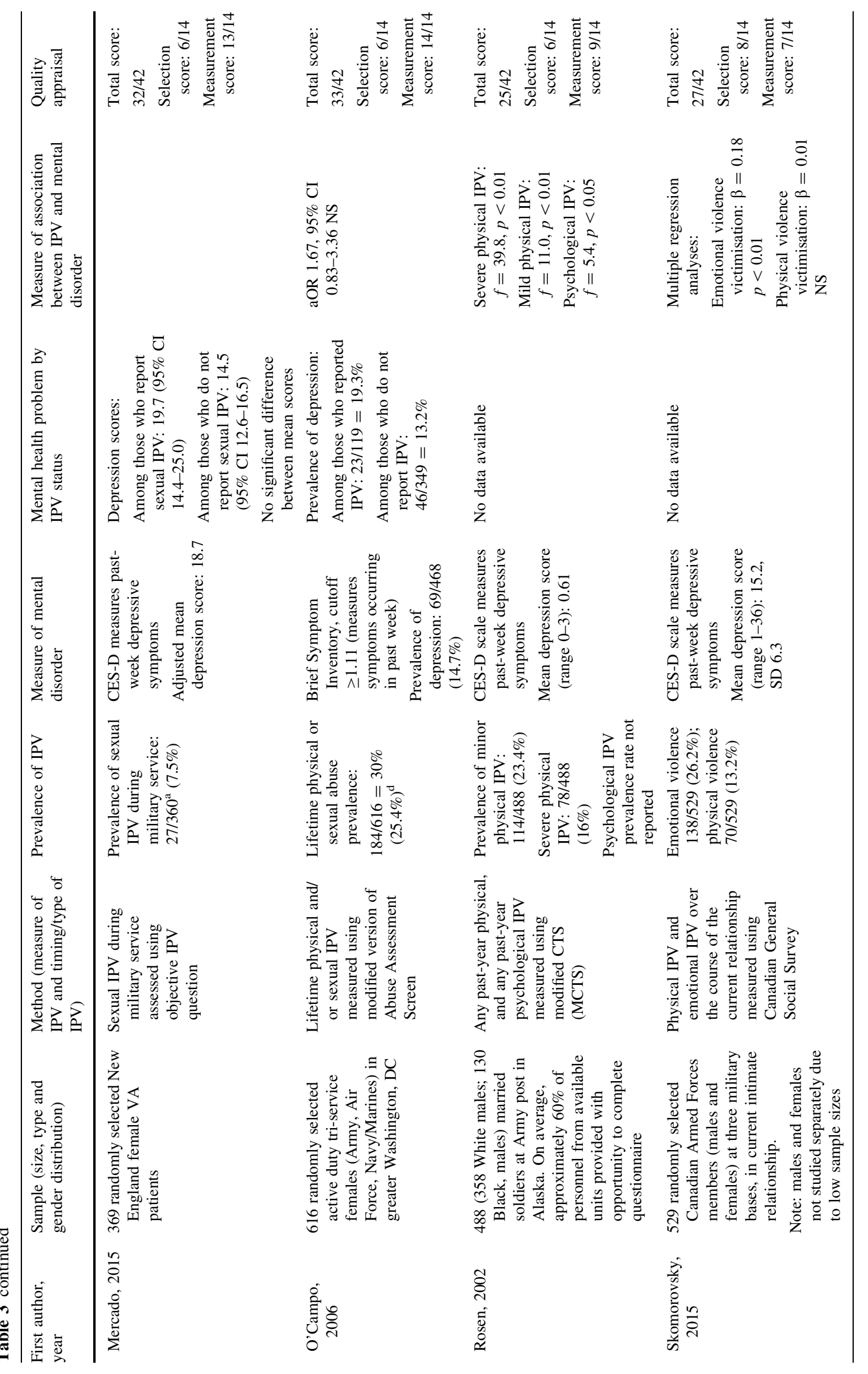




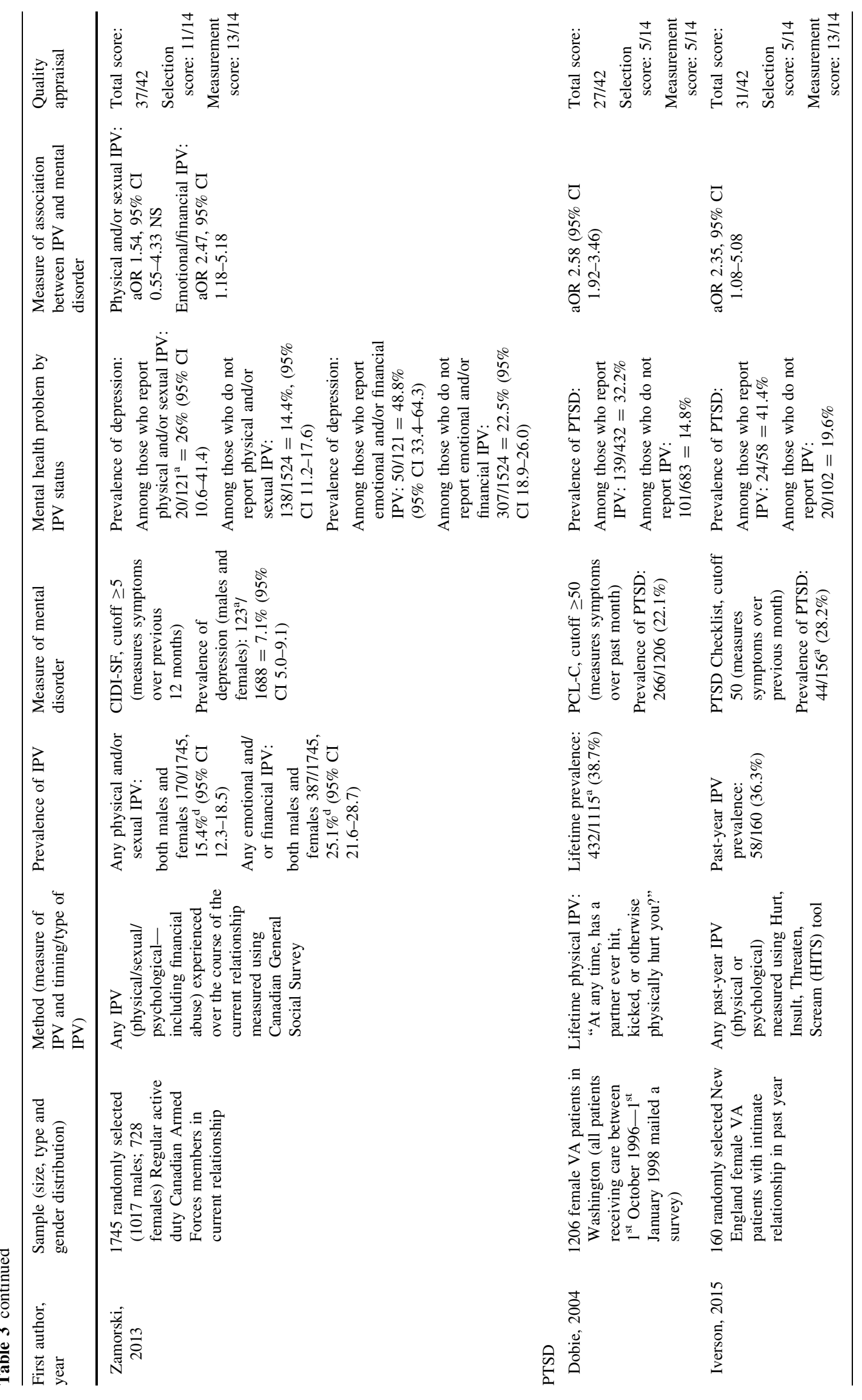




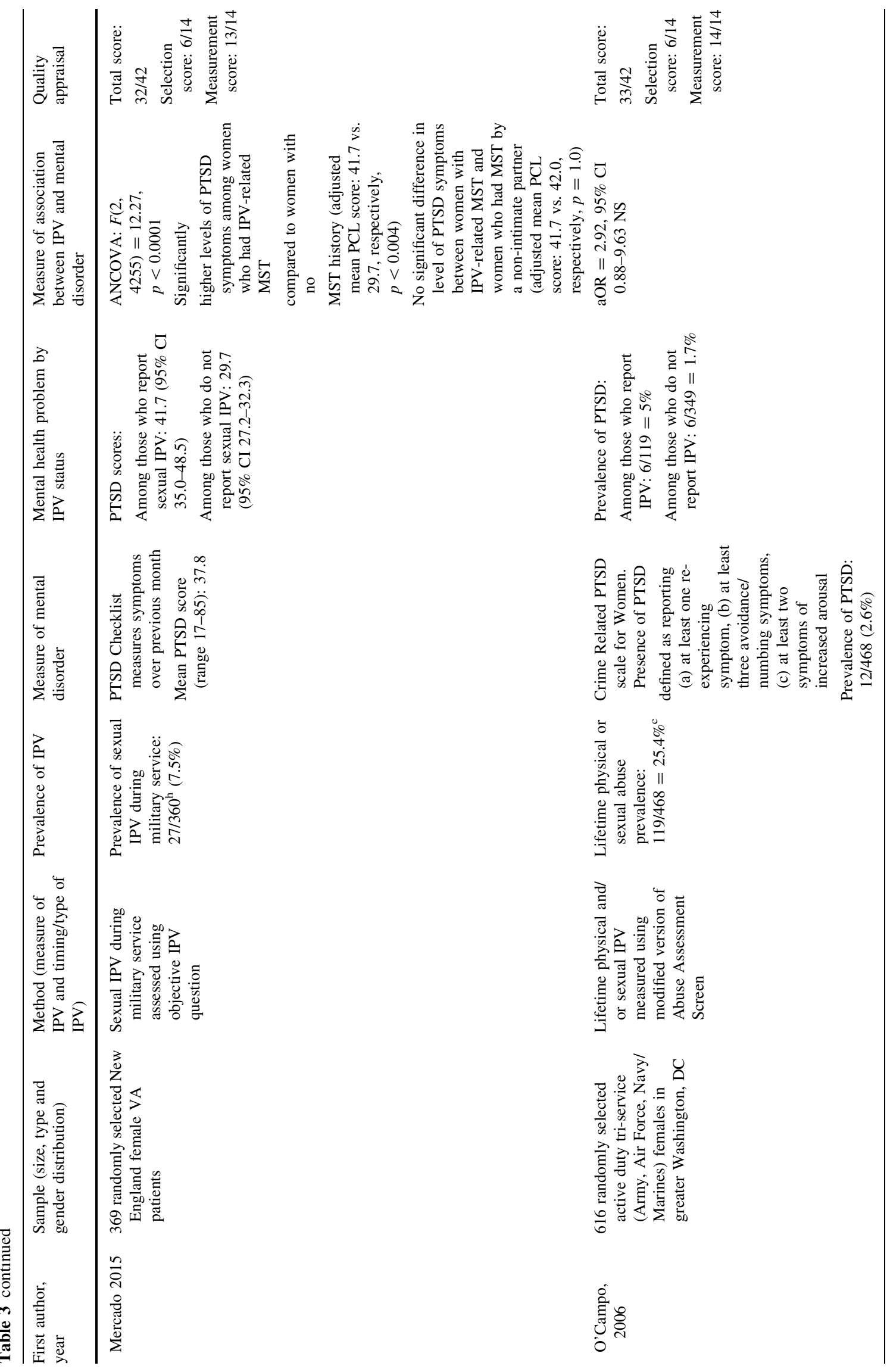




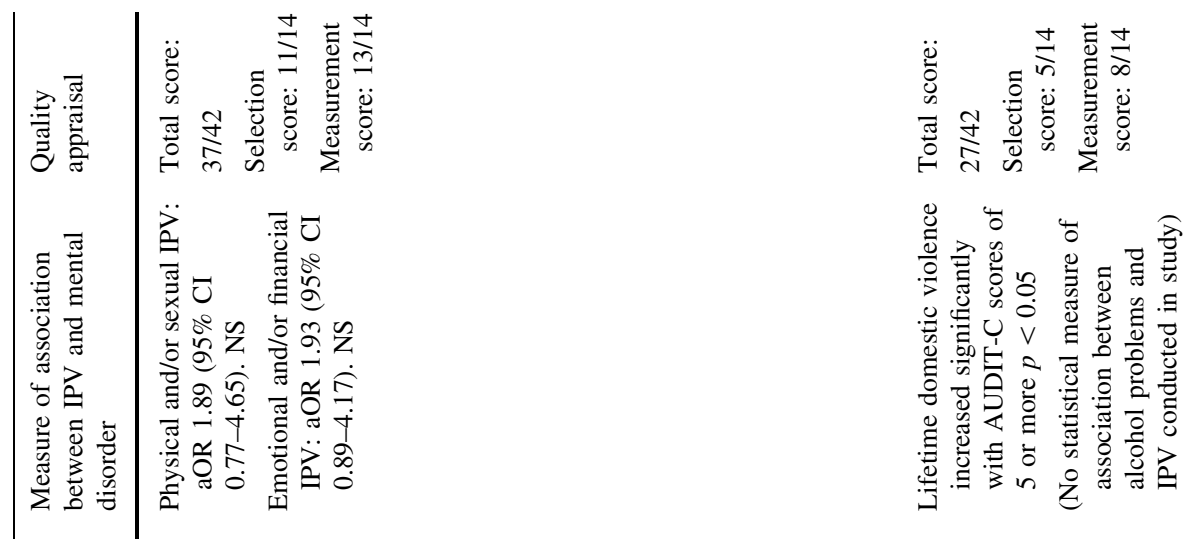

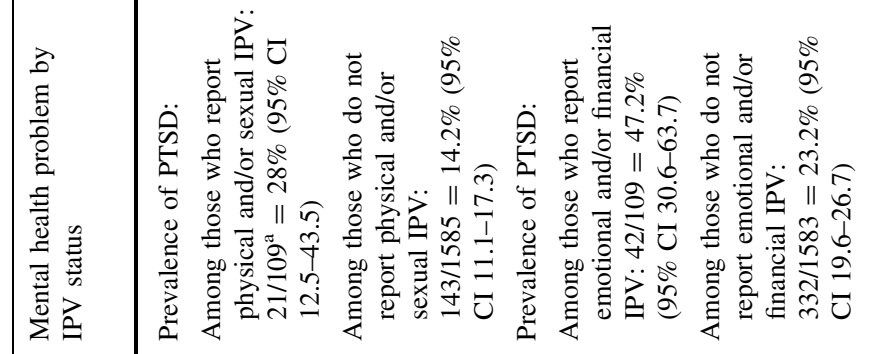

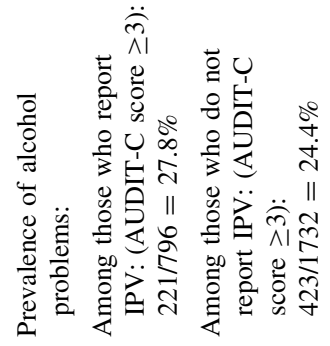

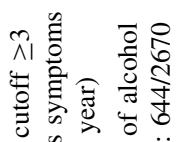

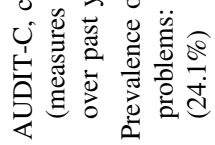

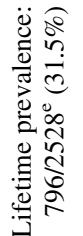

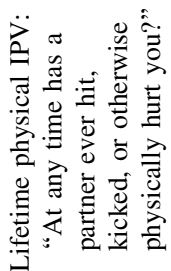

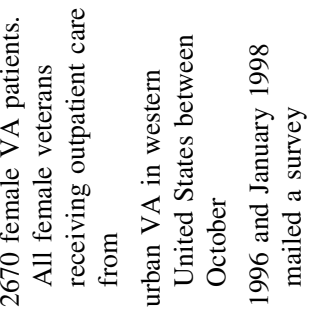

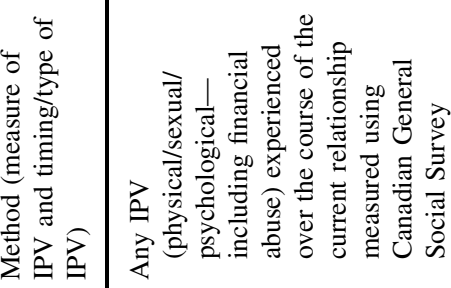

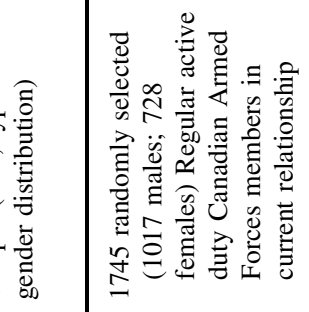

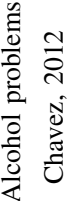

党 


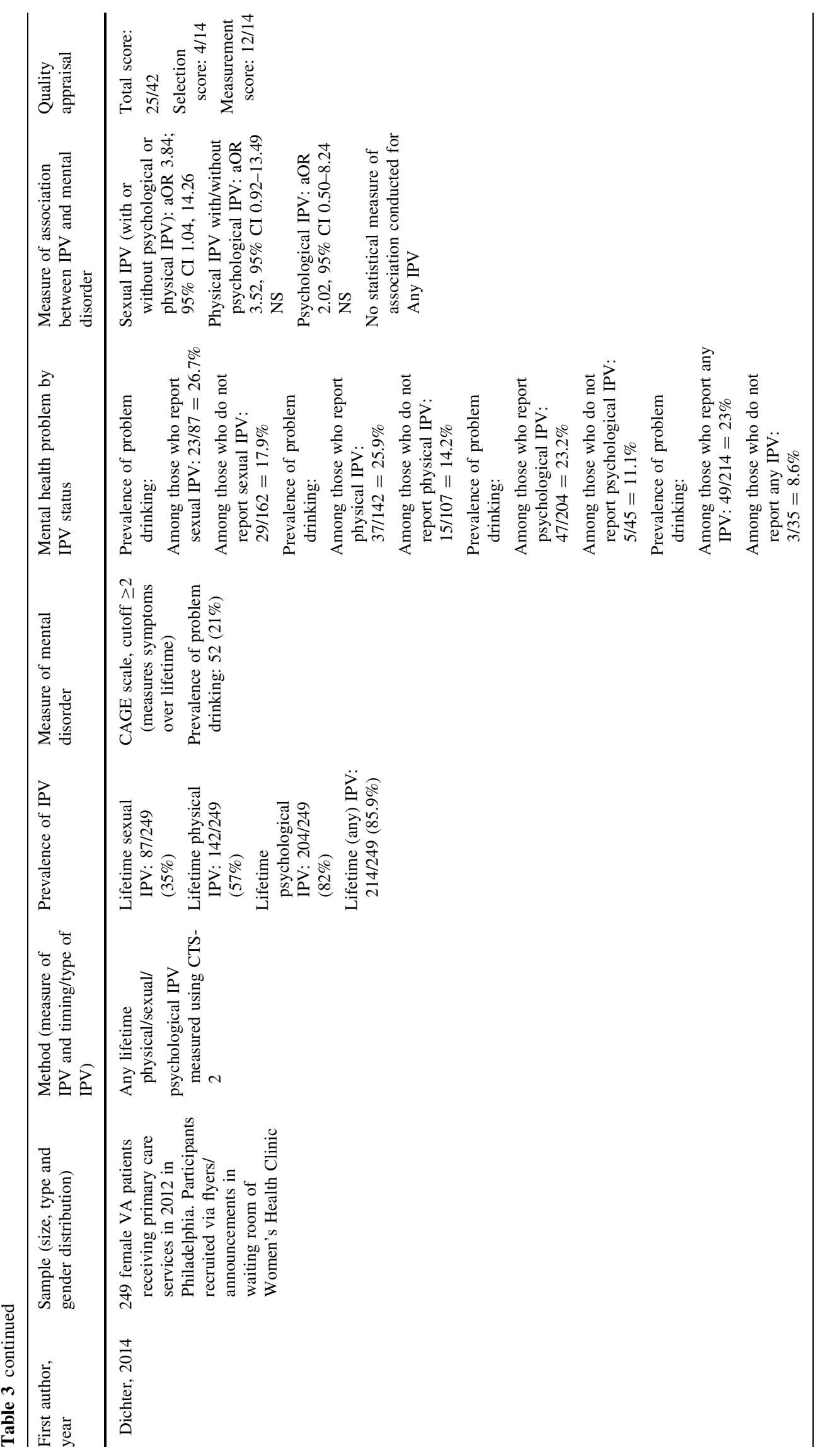




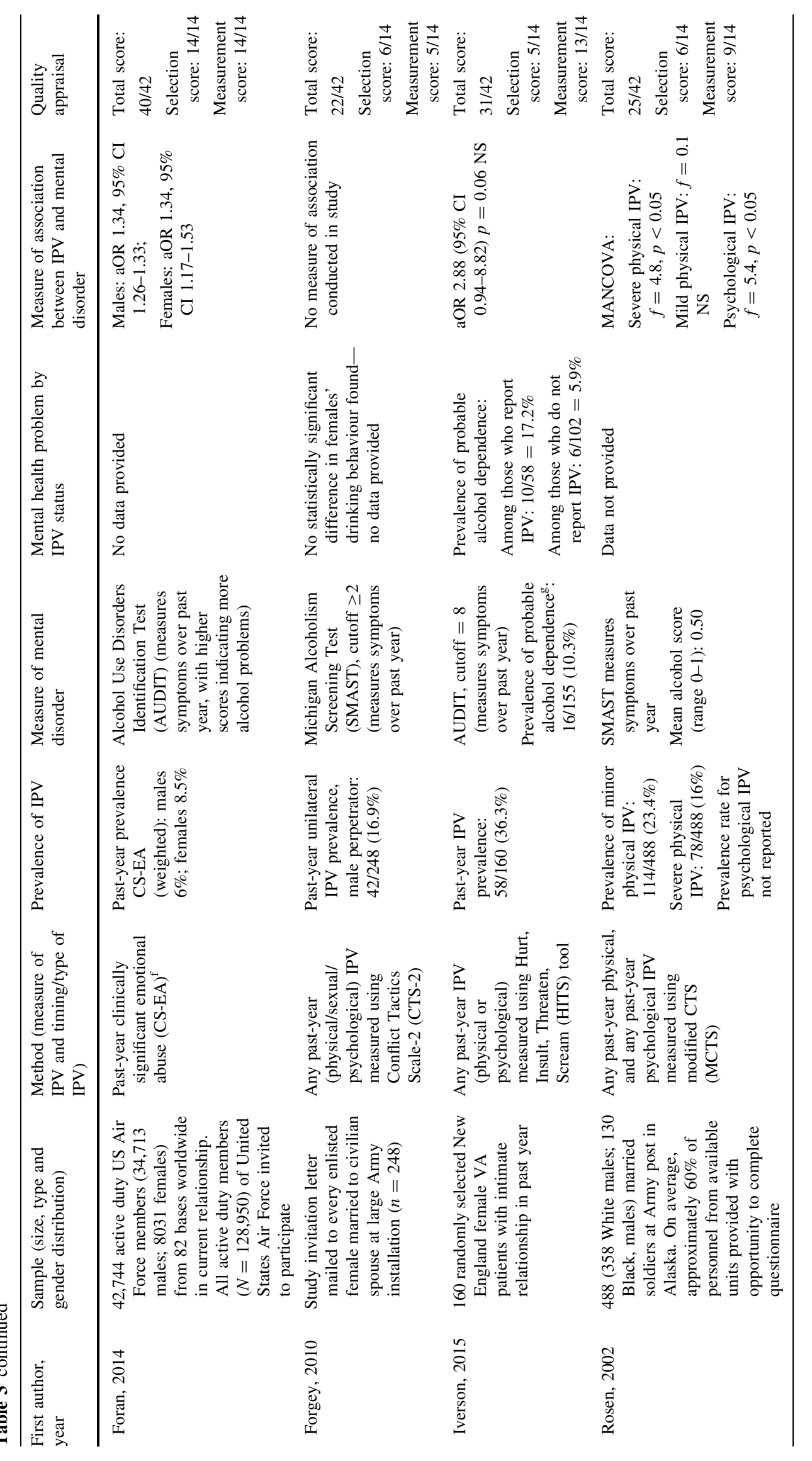




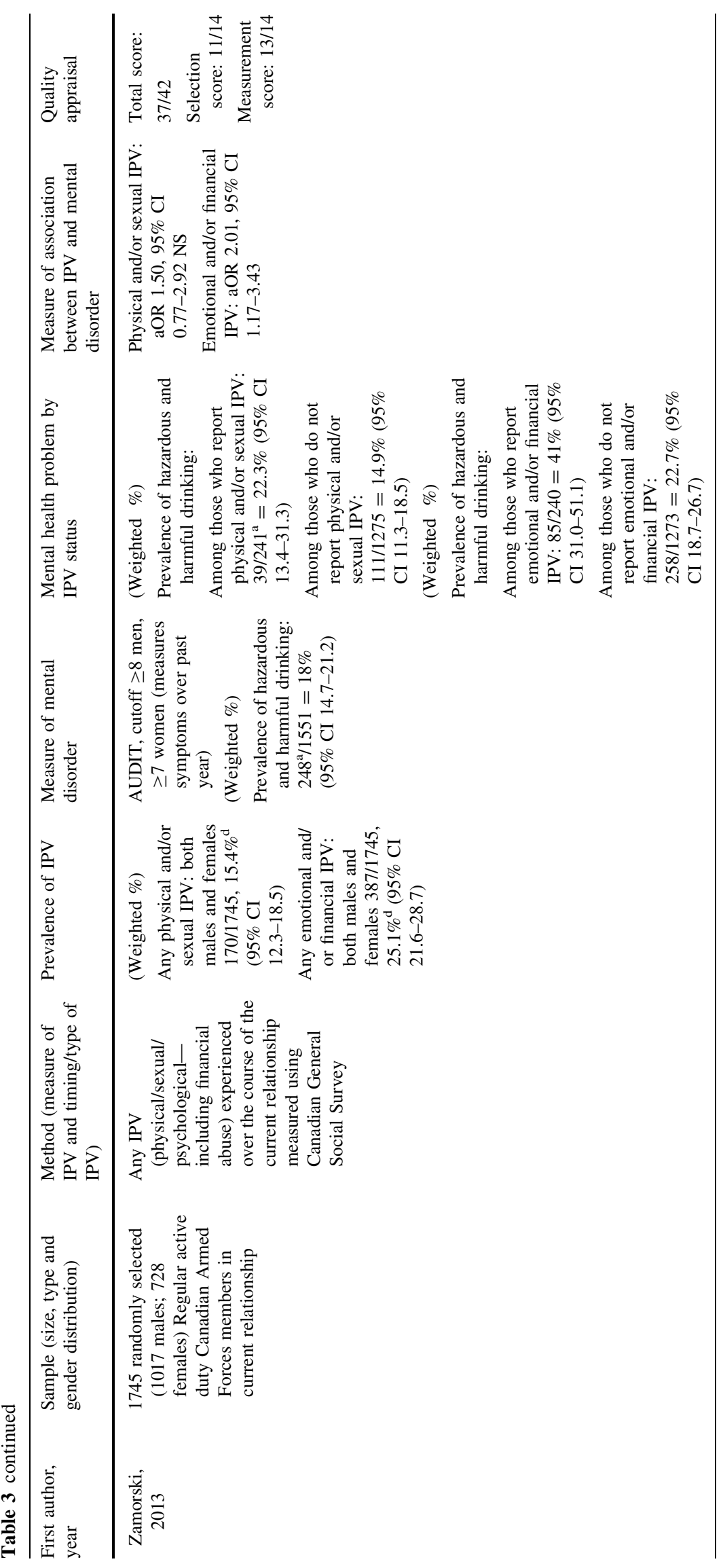




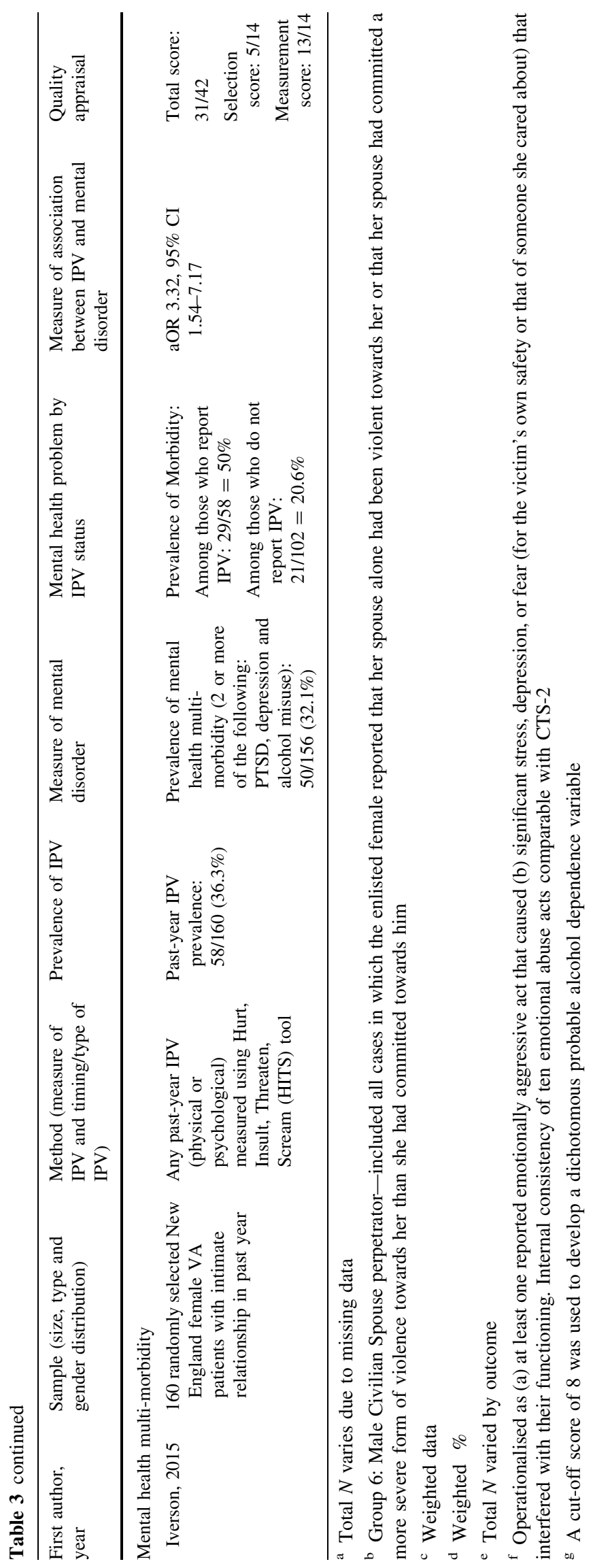


associated with depression/alcohol problems than physi$\mathrm{cal} / \mathrm{sexual} \mathrm{IPV.}$

\section{Link between IPV and mental disorder among military personnel}

Six of the seven studies that examined the association between IPV and depression found a significant association after controlling for potential confounders, with four studies being of high quality. All studies that explored psychological IPV and depression found a significant association [44, 45, 54]. Evidence for an association between physical and/or sexual IPV and depression was less consistent, with three out of four studies finding no significant association (two of which were high quality). Veteran studies tended not to look at IPV sub-types, but consistently found associations between IPV and depression. Of note, the two high-quality studies that reported on both males and females together found that depression was associated with psychological IPV, but not with physi$\mathrm{cal} / \mathrm{sexual}$ IPV [44, 45]. Overall, the number and quality of studies finding an association between IPV victimisation and depression were similar between males and females. A bi-directional causal relationship has been found between IPV and depression in the general population, though there are limited data on this relationship for men [86, 87]. A traumatic stress response framework has frequently been used to conceptualize the link between IPV and depression: traumatic events such as domestic abuse can cause fear, stress, and feelings of helplessness, isolation, and powerlessness, which may lead to depression [86, 88-92]. Salcioglu et al. [93] found that the strongest predictors of depression and PTSD in IPV survivors were helplessness and fear due to a sense of ongoing threat to safety. It has been suggested that a chronic traumatic stress response, where a victim is subjected to ongoing abuse, may lead to alterations in affect and sense of self (i.e., the predominance of self-blame and depressive affect) [94-96]. Common risk factors exist between IPV and depression, such as demographics, childhood adverse events, and substance use, which would need to be controlled for in research into this association [86].

Three of the five studies that examined the association between IPV and PTSD found a significant association after controlling for confounders. All three studies utilised female veteran samples, and none were rated as high quality. Two studies of active duty personnel (one was high quality) did not find a statistically significant association after adjustment for confounders [44, 58] (see Table 3). Only one study included male participants, but the sample was mixed gender and analyses were not stratified by gender. Therefore, it was not possible to comment on differences between males and females. There were too few studies to comment on differences in the association between sub-types of IPV and PTSD.

Four of the five studies (two were high quality) that investigated the association between IPV and alcohol problems found a significant association after controlling for potential confounders. An association between IPV and alcohol problems was more consistently found among male compared to female personnel in this review. However, it should be noted that the one high-quality study providing separate data on males and females reported identical odds ratios for emotional IPV and alcohol problems [50]. Among males and in active duty samples, psychological IPV was consistently found to be associated with alcohol problems [44, 50, 54]. Among females, the results were mixed. Only one veteran study explored the association between psychological IPV and alcohol problems, and no significant association was found. Overall, psychological IPV was more consistently found to be associated with alcohol problems than physical IPV.

The National Violence Against Women Survey (NVAWS) of males and females aged 18-65 found that psychological IPV was more strongly associated with adverse health outcomes (including depressive symptoms and substance use) than physical IPV [65]. The findings of the current review also support this: among active duty personnel, psychological IPV was more consistently associated with depression/alcohol problems than physi$\mathrm{cal} / \mathrm{sexual}$ IPV. Previous research has found that perpetrators of IPV are more likely to disclose psychological than physical abuse [97]. It is possible that a similar pattern is present among victims of IPV, though for perhaps different reasons. Wider research has found that barriers to the disclosure of IPV among mental health service users include fear of the consequences (including fear of Social Services involvement/child protection issues) and feelings of shame [98]. It is possible that victims perceive these barriers to disclosure to be greater in the context of physical/sexual than psychological violence.

\section{Veteran vs active duty}

Significant associations between IPV and depression/PTSD were more consistently found among veterans than active duty personnel. Research has confirmed the under-reporting of mental health problems among serving military personnel [99, 100]. Identified barriers to help-seeking include feared impact on an individual's military career [101-106] and also practical barriers such as lack of time due to a busy schedule $[100,105,107]$. A recent metaanalysis described the most frequently reported deterrents to seeking help for mental health problems; "My unit leadership might treat me differently" and "I would be seen as weak" [108]. Service providers working with 
military couples have observed that these barriers mean that personnel may be more open to seeking help after their military career has ended [109]. Furthermore, it has been found that the wives of active duty males refrained from disclosing IPV victimisation as they wanted to appear strong and capable to avoid being perceived as a "failed" military wife [110]. There is no similar research among samples of serving personnel who are victims of IPV, but such barriers to disclosure of abuse may exist.

Research is also emerging showing higher rates of mental health and social problems among veterans than among active duty personnel [41, 111]. A number of factors may contribute to this, such as the impact of transition [37, 112], loss of role or identity [30], and fragmentation of the social support network enjoyed in the military [113]. These factors could lead to the apparent increased association between IPV and mental health problems among veterans compared to active duty personnel.

The number and quality of studies finding an association between IPV and alcohol problems were found to be higher among active duty than veteran samples. There is a culture of excess alcohol consumption in the military [114]. It has been observed that military culture "fosters a warrior ethos that rewards physical and emotional prowess and frowns upon weakness and timidity" [115]. It is possible that active duty personnel perceive depression/PTSD to be more closely associated with weakness and, therefore, less acceptable than alcohol problems, thereby leading to the under-reporting of the latter. It is also possible that use of alcohol is a coping mechanism that masks the symptoms of other mental disorders. Alcohol misuse is highly comorbid with mental disorders such as depression and PTSD among military personnel [116].

\section{Impact of gender}

Perhaps, the most striking finding of this review was the lack of research into male IPV victimisation and mental health. Research in the general population has shown that women are at greater risk of IPV victimisation compared to men, and the psychiatric burden of IPV is greater among women [117]. Research into IPV and mental health among women in the military is, therefore, necessary. However, the National Violence Against Women Survey (NVAWS) found that, for both men and women, IPV victimisation was associated with increased risk of current poor health, depressive symptoms, substance use, chronic mental illness, chronic physical disease, and injury [65]. This is supported by further research in the general population that IPV can impact significantly on the psychological health of male victims [65, 118, 119]. The military culture, that favours male strength and is forbidding of male weakness, may have influenced the direction of research to focus on female IPV victims. However, it has been noted that to frame the problem as 'violence against women' overlooks males who may be victims of violence in gender-saturated contexts, such as IPV [120]. Walby et al. [121] argue that if the focus in official crime statistics is biased towards women, then we cannot explore the gendered nature of violence, which requires comparisons between males and females.

Studies in both the general population and military samples have found that men and women are equally likely to be violent in intimate relationships, but women are more likely to suffer an injury and are at greater risk of serious and sexual assaults [120, 122-128]. Not only were there too few studies of male victimisation to compare the impact of IPV on mental health by gender, but studies also neglected to measure impact of IPV. The only study that considered impact [85] found that in almost two-thirds of the cases of bi-directional violence of differing levels of severity ( $15.5 \%$ of all violence), the more severe violence was perpetrated by the male civilian spouse. A higher prevalence of injury was found among females (16.4\%) compared to males (11\%) [85]. However, it is important to note that in this study, enlisted females were asked to report on both their own and their spouse's behaviour, and therefore, there is likely to be significant reporting bias. Considering that, in the general population, the proportion of homicides committed by an intimate partner is six times higher for female $(38.6 \%)$ than for male $(6.3 \%)$ homicides [126], it seems that gender differences in IPV victimisation in this review may be masked by the lack of measurement of the impact of IPV. Walby et al. [121] assert that the gendered lack of alignment between actions and impact/consequences means that actions alone cannot be relied upon to define a violent event. Consequently, the authors argue that the CTS [129] is not an appropriate tool to measure violence, as it focuses on actions only and excludes impact/consequences, meaning that it is incompatible with the concept of crime used in criminal justice systems.

\section{Strengths and limitations}

To our knowledge, this is the first systematic review of studies of IPV victimisation and mental disorder among military populations. The strengths of this review are that it included studies of psychological and sexual IPV, rather than just physical violence, and it only included studies that used validated tools to measure symptoms of mental disorder. The interpretation of the review findings was limited by heterogeneity among the included studies. Diverse tools were used across studies to measure IPV and there were variations in the timing of IPV studied (for example, pastyear or lifetime, IPV experienced over the course of the 
current relationship, or during military service). These inconsistencies made comparisons between studies difficult.

Problems with IPV measurement were not only a significant limitation of all studies in this review, but also are a criticism of the field of IPV research as a whole [130-132]. IPV research has been based on varied and poorly-defined definitions of the types of IPV, particularly of "Any" IPV, hindering meaningful comparisons between studies [130]. Research findings are potentially distorted by reliance on participants' self-report on their partners' behaviour [130, 133, 134]. There is also no consensus as to whether threats of physical harm should be measured by physical abuse scales, or psychological abuse tools [135]. This is problematic considering that, not surprisingly, methods of IPV measurement have a powerful influence on study findings [131].

Only two studies in this review considered differences in the severity of IPV [54, 85]. It has been observed that combining individuals, who experience a high frequency of mild incidents with those experiencing a low frequency of severe violence, may result in distortions when making comparisons across research [130, 136]. It has been observed that IPV is often reciprocal and frequently occurs during interpersonal events. However, there is little acknowledgement of this in current methods of IPV measurement [137]. Only one study in this review considered whether victims also perpetrated violence, and found that over $60 \%$ of all reported violence was bi-directional [85]. The lack of consideration of patterns of violence between couples may have led to some misclassification bias among studies. IPV measures have been criticised for a lack of consideration of the context of abusive actions, for example, not excluding physically forceful acts that are used in self-defence [138]. However, there is no consensus on the specific contexts (e.g., retaliation) that should be examined to ensure accuracy of data collection [131]. Follingstad and colleagues suggest that continuing with the current approach to measuring IPV hinders the improvement of the current evidence base, and stresses the importance of developing a "gold standard" measurement that would allow for meaningful comparison of research findings [131].

All studies included in this review used validated tools to measure mental disorder. However, some measured symptoms rather than providing a diagnosis, limiting the reliability and comparability of study findings. Studies did not consistently control for potential confounders when examining the association between IPV and mental disorder. Finally, all included studies were cross sectional, meaning that no conclusions can be drawn regarding the direction of causality between IPV victimisation and mental disorders.

\section{Implications}

The findings from this review indicate that, just like among civilian populations [65], the burden of mental health need may be significant among military personnel who are victims of IPV. This emphasises the important role of health as well as welfare workers in the identification and management of IPV and its consequences. We need research to help us better understand barriers to the reporting of IPV in military culture, in order that effective interventions can be developed.

IPV is associated with adverse health consequences for both male and female victims [65], and there is considerable evidence that men are less likely than women to seek help for diverse mental and physical health problems [139]. In the UK, Joint Service Publication (JSP) policies detailing procedures for military welfare provision surrounding IPV have been developed based on the Ministry of Defence's commitment to support the cross government Violence Against Women and Girls agenda [140]. Notably, although the JSP policy acknowledges the possibility of male victimisation in its definition of IPV, the sections providing practice direction for IPV cases and detailing safety planning procedures focus on the victim being female [141]. This is suggestive of a lack of focus on male victimisation, which may be exacerbated by persistent attitudes towards gender roles in military culture and is supported by the lack of research on males in this review. IPV awareness and management is more advanced in the US military, most likely driven by the larger body of research literature (all studies in this review were based in the USA or Canada), where there is greater emphasis on prevention strategies [142] and they differentiate between civilian and serving victims [143]. In the US, victim advocate services and the Family Advocacy Program are widespread [143]. Domestic violence advocacy has been introduced in the UK in recent years [66, 70]; however, research trialling these methods in military environments needs to be conducted, as it has been in the US [144].

This review highlights the need for further research to examine IPV victimisation and mental disorder among active duty and veteran military personnel. There is a need for greater consistency in IPV measurement to allow meta-analyses of the findings of different studies. Future research should consider the impact of IPV victimisation in order that gender differences can be better understood.

\section{Compliance with ethical standards}

Conflict of interest On behalf of all authors, the corresponding author states that there is no conflict of interest. 
Open Access This article is distributed under the terms of the Creative Commons Attribution 4.0 International License (http://crea tivecommons.org/licenses/by/4.0/), which permits unrestricted use, distribution, and reproduction in any medium, provided you give appropriate credit to the original author(s) and the source, provide a link to the Creative Commons license, and indicate if changes were made.

\section{References}

1. World Health Organization (2012) Understanding and addressing violence against women: intimate partner violence. World Health Organization, Geneva, Switzerland

2. Centers for Disease Control and Prevention (n.d.) National Center for Injury Prevention \& Control: Division of Violence Prevention. https://www.cdc.gov/violenceprevention/intimate partnerviolence/index.html. Accessed 2 Nov 2016

3. Devries KM, Mak JY, García-Moreno C, Petzold M, Child JC, Falder G, Lim S, Bacchus LJ, Engell RE, Rosenfeld L (2013) The global prevalence of intimate partner violence against women. Science 340(6140):1527-1528

4. Vos T, Astbury J, Piers L, Magnus A, Heenan M, Stanley L, Walker L, Webster K (2006) Measuring the impact of intimate partner violence on the health of women in Victoria, Australia. Bull World Health Organ 84(9):739-744

5. Marshall AD, Panuzio J, Taft CT (2005) Intimate partner violence among military veterans and active duty servicemen. Clin Psychol Rev 25(7):862-876

6. Jones AD (2012) Intimate partner violence in military couples: a review of the literature. Aggress Violent Beh 17(2):147-157

7. Rentz ED, Martin SL, Gibbs DA, Clinton-Sherrod M, Hardison J, Marshall SW (2006) Family violence in the military a review of the literature. Trauma Violence Abuse 7(2):93-108

8. Butterfield F (2002) Wife killings at fort reflect growing problem in military. The New York Times. http://www.nytimes.com/ 2002/07/29/us/wife-killings-at-fort-reflect-growing-problem-inmilitary.html. Accessed 18 Oct 2016

9. Clark JC, Messer SC (2006) Intimate partner violence in the US military: rates, risks, and responses. In: Castro $\mathrm{CA}$, Adler $\mathrm{AB}$, Britt TW (eds) The military life. Military life: the psychology of serving in peace and combat: the military family. Praeger Security International, Westport, CT, pp 193-219

10. French C, Dandeker C (2005) UK military families and the deployments to Iraq: preliminary findings from a pre-, during-, and post-deployment study of the British Army. In: InterUniversity Seminar on Armed Forces and Society, Chicago, USA

11. MacManus D, Dean K, Al Bakir M, Iversen AC, Hull L, Fahy T, Wessely S, Fear NT (2012) Violent behaviour in UK military personnel returning home after deployment. Psychol Med 42(8): $1663-1673$

12. MacManus D, Dean K, Jones M, Rona RJ, Greenberg N, Hull L, Fahy T, Wessely S, Fear NT (2013) Violent offending by UK military personnel deployed to Iraq and Afghanistan: a data linkage cohort study. Lancet 381(9870):907-917

13. MacManus D, Rona R, Dickson H, Somaini G, Fear N, Wessely S (2015) Aggressive and violent behavior among military personnel deployed to Iraq and Afghanistan: prevalence and link with deployment and combat exposure. Epidemiol Rev 37(1):196-212

14. Kulka RA, Schlenger WE, Fairbank JA, Hough RL, Jordan BK, Marmar CR, Weiss DS (1988) Contractual report of findings from the National Vietnam veterans readjustment study. Research Triangle Institute, Research Triangle Park
15. Lazar SG (2014) The mental health needs of military service members and veterans. Psychodyn Psychiatry 42(3):459-478

16. Sayers SL, Farrow VA, Ross J, Oslin DW (2009) Family problems among recently returned military veterans referred for a mental health evaluation. J Clin Psychiatry 70(2):163

17. Burrell LM, Adams GA, Durand DB, Castro CA (2006) The impact of military lifestyle demands on well-being, army, and family outcomes. Armed Forces Soc 33(1):43-58

18. Williamson E (2012) Domestic abuse and military families: the problem of reintegration and control. $\mathrm{Br} \mathrm{J}$ Soc Work 42(7):1371-1387

19. Samele C (2013) The mental health of serving and exService personnel. https://www.mentalhealth.org.uk/sites/ default/files/the-mental-health-of-serving-and-ex-service-per sonnel.pdf. Accessed 18 Nov 2016

20. Burland D, Lundquist JH (2013) "The best years of our lives": military service and family relationships-a life course perspective. In: Wilmouth JM, London AS (eds) Life course perspectives on military service. Routledge, New York, NY

21. Diehle J, Brooks SK, Greenberg N (2017) Veterans are not the only ones suffering from posttraumatic stress symptoms: what do we know about dependents' secondary traumatic stress? Soc Psychiatry Psychiatr Epidemiol 52(1):1-10

22. Galovski T, Lyons JA (2004) Psychological sequelae of combat violence: a review of the impact of PTSD on the veteran's family and possible interventions. Aggress Violent Beh 9(5):477-501

23. Gimbel C, Booth A (1994) Why does military combat experience adversely affect marital relations? J Marriage Fam 56(3):691-703

24. Monson CM, Taft CT, Fredman SJ (2009) Military-related PTSD and intimate relationships: from description to theorydriven research and intervention development. Clin Psychol Rev 29(8):707-714

25. Murphy D, Palmer E, Busuttil W (2016) Mental health difficulties and help-seeking beliefs within a sample of female partners of UK veterans diagnosed with post-traumatic stress disorder. J Clin Med 5(8):68

26. Orcutt HK, King LA, King DW (2003) Male-perpetrated violence among Vietnam veteran couples: relationships with veteran's early life characteristics, trauma history, and PTSD symptomatology. J Trauma Stress 16(4):381-390

27. Riggs DS, Byrne CA, Weathers FW, Litz BT (1998) The quality of the intimate relationships of male Vietnam veterans: problems associated with posttraumatic stress disorder. J Trauma Stress 11(1):87-101

28. Savarese VW, Suvak MK, King LA, King DW (2001) Relationships among alcohol use, hyperarousal, and marital abuse and violence in Vietnam veterans. J Trauma Stress 14(4):717-732

29. Solomon Z, Dekel R, Zerach G (2008) The relationships between posttraumatic stress symptom clusters and marital intimacy among war veterans. J Fam Psychol 22(5):659

30. Binks E, Cambridge S (2017) The transition experiences of british military veterans. Polit Psychol. doi:10.1111/pops.12399

31. Dandeker C, Wessely S, Iversen A, Ross J (2003) Improving the delivery of cross departmental support and services for veterans. King's College London, Deparment of War Studies and Institute of Psychiatry, London

32. Iversen A, Dyson C, Smith N, Greenberg N, Walwyn R, Unwin C, Hull L, Hotopf M, Dandeker C, Ross J (2005) 'Goodbye and good luck': the mental health needs and treatment experiences of British ex-service personnel. Br J Psychiatry 186(6):480-486

33. Lord Ashcroft (2014) The veterans' transition review. http:// www.veteranstransition.co.uk/vtrreport.pdf. Accessed 25 May 2017 
34. Mansfield AJ, Bender RH, Hourani LL, Larson GE (2011) Suicidal or self-harming ideation in military personnel transitioning to civilian life. Suicide and life-threatening behavior 41(4):392-405

35. Thompson J, Hopman W, Sweet J, VanTil L, MacLean MB, VanDenKerkhof E, Sudom K, Poirier A, Pedlar D (2013) Health-related quality of life of Canadian Forces veterans after transition to civilian life. Can J Public Health 104(1):e15-e21

36. Iversen AC, van Staden L, Hughes JH, Browne T, Hull L, Hall J, Greenberg N, Rona RJ, Hotopf M, Wessely S (2009) The prevalence of common mental disorders and PTSD in the UK military: using data from a clinical interview-based study. BMC Psychiatry 9(1):68

37. Hatch SL, Harvey SB, Dandeker C, Burdett H, Greenberg N, Fear NT, Wessely S (2013) Life in and after the Armed Forces: social networks and mental health in the UK military. Sociol Health Illn 35(7):1045-1064

38. Sher L, Braquehais MD, Casas M (2012) Posttraumatic stress disorder, depression, and suicide in veterans. Cleve Clin J Med 79(2):92-97

39. Hoglund MW (2014) Mental health in deployed and nondeployed veteran men and women in comparison with their civilian counterparts. Mil Med 179(1):19-25

40. Cameron M, Wan CKV, Vu AV, Woo DT, Lie JD, Tu KN (2016) Suicide in the veteran population. US Pharm 41(11):HS12-HS18

41. Hoerster KD, Lehavot K, Simpson T, McFall M, Reiber G, Nelson KM (2012) Health and health behavior differences: U.S. military, veteran, and civilian men. Am J Prev Med 43(5):483-489

42. Pengpid S, Peltzer K (2016) Intimate partner violence victimization and associated factors among male and female university students in 22 countries in Africa, Asia and the Americas. Afr J Reprod Health 20(1):29-39

43. Smith Slep AM, Foran HM, Heyman RE, Snarr JD (2011) Risk factors for clinically significant intimate partner violence among active-duty members. J Marriage Fam 73(2):486-501. doi:10. 1111/j.1741-3737.2010.00820.x. https://www.scopus.com/ inward/record.uri?eid=2-s2.0-79952508780\&doi $=10.1111 \% 2 \mathrm{fj}$. 1741-3737.2010.00820.x\&partnerID=40\&md5= 5088e80e478cf3a46bca27f2ca684a40

44. Zamorski MA, Wiens-Kinkaid ME (2013) Cross-sectional prevalence survey of intimate partner violence perpetration and victimization in Canadian military personnel. BMC Public Health 13(1):1019

45. Skomorovsky A, Hujaleh F, Wolejszo S (2015) Intimate partner violence in the Canadian armed forces: the role of family stress and its impact on well-being. Mil Med 180(7):809-816. doi:10. 7205/milmed-d-14-00447

46. Heyman RE, Neidig PH (1999) A comparison of spousal aggression prevalence rates in US Army and civilian representative samples. J Consult Clin Psychol 67(2):239

47. Dichter ME, Cerulli C, Bossarte RM (2011) Intimate partner violence victimization among women veterans and associated heart health risks. Womens Health Issues 21(4):S190-S194. doi: $10.1016 /$ j.whi.2011.04.008

48. Cerulli C, Bossarte RM, Dichter ME (2014) Exploring intimate partner violence status among male veterans and associated health outcomes. Am J Mens Health 8(1):66-73. doi:10.1177/ 1557988313492558

49. Belik S-L, Stein MB, Asmundson GJ, Sareen J (2009) Relation between traumatic events and suicide attempts in Canadian military personnel. Can J Psychiatry 54(2):93-104

50. Foran HM, Heyman RE, Slep AMS, Res USAFFA (2014) emotional abuse and its unique ecological correlates among military personnel and spouses. Psychol Violence 4(2):128-142. doi:10.1037/a0034536
51. Forgey MA, Badger L (2006) Patterns of intimate partner violence among married women in the military: type, level, directionality and consequences. J Fam Violence 21(6):369-380. doi:10.1007/s10896-006-9033-3

52. Mercado R, Foynes MM, Carpenter SL, Iverson KM (2015) Sexual Intimate partner violence as a form of MST: an initial investigation. Psychol Serv 12(4):348-356. doi:10.1037/ ser0000056

53. Murdoch M, Nichol KL (1995) Women veterans' experiences with domestic violence and with sexual harassment while in the military. Arch Fam Med 4(5):411-418

54. Rosen LN, Parmley AM, Knudson KH, Fancher P (2002) Intimate partner violence among married male U.S. army soldiers: ethnicity as a factor in self-reported perpetration and victimization. Violence Vict 17(5):607-622

55. Rosen LN, Parmley AM, Knudson KH, Fancher P (2002) Gender differences in the experience of intimate partner violence among active duty U.S. Army soldiers. Mil Med 167(12):959-963

56. Iverson KM, Pogoda TK (2015) Traumatic brain injury among women veterans: an invisible wound of intimate partner violence. Med Care 53:S112-S119

57. Lutgendorf MA, Busch JM, Doherty DA, Conza LA, Moone SO, Magann EF (2009) Prevalence of domestic violence in a pregnant military population. Obstet Gynecol 113(4):866-872

58. O'Campo P, Kub J, Woods A, Garza M, Jones AS, Gielen AC, Dienemann J, Campbell J (2006) Depression, PTSD, and comorbidity related to intimate partner violence in civilian and military women. Brief Treat Crisis Interv 6(2):99-110. doi:10. 1093/brief-treatment/mhj010

59. Dichter ME, Marcus SC (2013) Intimate partner violence victimization among women veterans: health, health care service use, and opportunities for intervention. Milit Behav Health 1(2):107-113. doi:10.1080/21635781.2013.830062

60. Cronin C (1995) Adolescent reports of parental spousal violence in military and civilian families. $\mathrm{J}$ Interpers Violence 10(1):117-122

61. Griffin WA, Morgan AR (1988) Conflict in maritally distressed military couples. Am J Fam Ther 16(1):14-22

62. Bradley C (2007) Veteran status and marital aggression: does military service make a difference? J Fam Violence 22(4):197-209. doi:10.1007/s10896-007-9072-4

63. Bundock L, Howard LM, Trevillion K, Malcolm E, Feder G, Oram S (2013) Prevalence and risk of experiences of intimate partner violence among people with eating disorders: a systematic review. J Psychiatr Res 47(9):1134-1142

64. Campbell JC (2002) Health consequences of intimate partner violence. The Lancet 359(9314):1331-1336

65. Coker AL, Davis KE, Arias I, Desai S, Sanderson M, Brandt HM, Smith PH (2002) Physical and mental health effects of intimate partner violence for men and women. Am J Prev Med 23(4):260-268

66. Flach C, Leese M, Heron J, Evans J, Feder G, Sharp D, Howard L (2011) Antenatal domestic violence, maternal mental health and subsequent child behaviour: a cohort study. BJOG Int J Obstetr Gynaecol 118(11):1383-1391

67. Golding JM (1999) Intimate partner violence as a risk factor for mental disorders: a meta-analysis. J Fam Violence 14(2):99-132

68. Howard LM, Oram S, Galley H, Trevillion K, Feder G (2013) Domestic violence and perinatal mental disorders: a systematic review and meta-analysis. PLoS Med 10(5):e1001452

69. Oram S, Trevillion K, Feder G, Howard L (2013) Prevalence of experiences of domestic violence among psychiatric patients: systematic review. Br J Psychiatry 202(2):94-99

70. Oram S, Trevillion K, Khalifeh H, Feder G, Howard L (2014) Systematic review and meta-analysis of psychiatric disorder and 
the perpetration of partner violence. Epidemiol Psychiatr Sci 23(04):361-376

71. Trevillion K, Oram S, Feder G, Howard LM (2012) Experiences of domestic violence and mental disorders: a systematic review and meta-analysis. PLoS One 7(12):e51740

72. Khalifeh H, Oram S, Trevillion K, Johnson S, Howard LM (2015) Recent intimate partner violence among people with chronic mental illness: findings from a national cross-sectional survey. Br J Psychiatry 207(3):207-212

73. Howard L, Trevillion K, Khalifeh H, Woodall A, Agnew-Davies R, Feder G (2010) Domestic violence and severe psychiatric disorders: prevalence and interventions. Psychol Med 40(06):881-893

74. Trevillion K, Williamson E, Thandi G, Borschmann R, Oram S, Howard LM (2015) A systematic review of mental disorders and perpetration of domestic violence among military populations. Soc Psychiatry Psychiatr Epidemiol 50(9):1329-1346

75. Office Home (2005) Domestic violence: a national report. Home Office, London

76. Downs SH, Black N (1998) The feasibility of creating a checklist for the assessment of the methodological quality both of randomised and non-randomised studies of health care interventions. J Epidemiol Community Health 52(6):377-384

77. Loney PL, Chambers LW (1998) Critical appraisal of the health research literature: prevalence or incidence of a health problem. Chronic Dis Can 19(4):170-177

78. Saha S, Chant D, Welham J, McGrath J (2005) A systematic review of the prevalence of schizophrenia. PLoS Med 2(5):e141

79. Wing JK (1994) The schedules for clinical assessment in neuropsychiatry. World Health Organization-Division of Mental Health \& Social Work, Geneva

80. CASP (2017) Critical appraisal skills programme (CASP): making sense of evidence. http://www.casp-uk.net/casp-toolschecklists. Accessed 4 June 2016

81. Chavez LJ, Williams EC, Lapham G, Bradley KA (2012) Association between alcohol screening scores and alcohol-related risks among female Veterans Affairs patients. J Stud Alcohol Drugs 73(3):391-400

82. Dichter ME, Marcus SC, Wagner C, Bonomi AE (2014) Associations between psychological, physical, and sexual intimate partner violence and health outcomes among women veteran VA patients. Soc Work Mental Health 12(5-6):411-428. doi:10. 1080/15332985.2013.870104

83. Dobie DJ, Kivlahan DR, Maynard C, Bush KR, Davis TM, Bradley KA (2004) Posttraumatic stress disorder in female veterans: association with self-reported health problems and functional impairment. Arch Intern Med 164(4):394-400

84. Iverson KM, Vogt D, Dichter ME, Carpenter SL, Kimerling R, Street AE, Gerber MR (2015) Intimate partner violence and current mental health needs among female veterans. J Am Board Fam Med 28(6):772-776

85. Forgey MA, Badger L (2010) Patterns of intimate partner violence and associated risk factors among married enlisted female soldiers. Violence Vict 25(1):45-61. doi:10.1891/0886-6708.25. 1.45

86. Devries KM, Mak JY, Bacchus LJ, Child JC, Falder G, Petzold M, Astbury J, Watts CH (2013) Intimate partner violence and incident depressive symptoms and suicide attempts: a systematic review of longitudinal studies. PLoS Med 10(5):e1001439

87. Hamberger LK, Saunders DG, Hovey M (1991) Prevalence of domestic violence in community practice and rate of physician inquiry. Fam Med 24(4):283-287

88. Beydoun HA, Beydoun MA, Kaufman JS, Lo B, Zonderman AB (2012) Intimate partner violence against adult women and its association with major depressive disorder, depressive symptoms and postpartum depression: a systematic review and metaanalysis. Soc Sci Med 75(6):959-975
89. Hyde JS, Mezulis AH, Abramson LY (2008) The ABCs of depression: integrating affective, biological, and cognitive models to explain the emergence of the gender difference in depression. Psychol Rev 115(2):291

90. Campbell JC, Lewandowski LA (1997) Mental and physical health effects of intimate partner violence on women and children. Psychiatr Clin North Am 20(2):353-374

91. Dutton MA (1992) Understanding women's responses to domestic violence: a redefinition of battered woman syndrome. Hofstra L Rev 21:1191

92. Filson J, Ulloa E, Runfola C, Hokoda A (2010) Does powerlessness explain the relationship between intimate partner violence and depression? J Interpers Violence 25(3):400-415

93. Salcioglu E, Urhan S, Pirinccioglu T, Aydin S (2017) Anticipatory fear and helplessness predict PTSD and depression in domestic violence survivors. Psychol Trauma Theory Res Pract Policy 9(1):117-125

94. Campbell J, Humphreys J (1993) Nursing care of survivors of family violence. Mosby Incorporated, Louis, MO

95. Dutton MA (2000) Empowering and healing the battered woman: a model for assessment and intervention. Springer Publishing Company, New York, NY

96. Herman JL (1997) Trauma and recovery, vol 551. Basic Books, New York, NY

97. Williamson E, Jones S, Hester M, Feder G (2014) Asking men about domestic violence and abuse (DVA) in a GP setting: Recruitment and participation. Univ Bristol, Bristol

98. Rose D, Trevillion K, Woodall A, Morgan C, Feder G, Howard L (2010) Barriers and facilitators of disclosures of domestic violence by mental health service users: qualitative study. Br J Psychiatry. doi:10.1192/bjp-bp.109.072389

99. Iversen AC, van Staden L, Hughes JH, Browne T, Greenberg N, Hotopf M, Rona RJ, Wessely S, Thornicroft G, Fear NT (2010) Help-seeking and receipt of treatment among UK service personnel. Br J Psychiatry 197(2):149-155

100. Hoge CW, Castro CA, Messer SC, McGurk D, Cotting DI, Koffman RL (2004) Combat duty in Iraq and Afghanistan, mental health problems, and barriers to care. N Engl J Med 351(1):13-22

101. Adler AB, Britt TW, Riviere LA, Kim PY, Thomas JL (2015) Longitudinal determinants of mental health treatment-seeking by US soldiers. Br J Psychiatry 207(4):346-350

102. Britt TW, Bennett EA, Crabtree M, Haugh C, Oliver K, McFadden A, Pury CL (2011) The theory of planned behavior and reserve component veteran treatment seeking. Milit Psychol 23(1):82

103. Britt TW, Jennings KS, Cheung JH, Pury CLS, Zinzow HM (2015) The role of different stigma perceptions in treatment seeking and dropout among active duty military personnel. Psychiatr Rehabil J 38(2):142-149. doi:10.1037/prj0000120

104. Kehle SM, Polusny MA, Murdoch M, Erbes CR, Arbisi PA, Thuras P, Meis LA (2010) Early mental health treatment-seeking among US National Guard soldiers deployed to Iraq. J Trauma Stress 23(1):33-40

105. Kim PY, Britt TW, Klocko RP, Riviere LA, Adler AB (2011) Stigma, negative attitudes about treatment, and utilization of mental health care among soldiers. Milit Psychol 23(1):65

106. Skopp NA, Bush NE, Vogel DL, Wade NG, Sirotin AP, McCann RA, Metzger-Abamukong MJ (2012) Development and initial testing of a measure of public and self-stigma in the military. J Clin Psychol 68(9):1036-1047

107. Britt TW, Greene-Shortridge TM, Brink S, Nguyen QB, Rath J, Cox AL, Hoge CW, Castro CA (2008) Perceived stigma and barriers to care for psychological treatment: implications for reactions to stressors in different contexts. J Soc Clin Psychol 27(4):317-335 
108. Sharp ML, Fear NT, Rona RJ, Wessely S, Greenberg N, Jones N, Goodwin L (2015) Stigma as a barrier to seeking health care among military personnel with mental health problems. Epidemiol Rev 37(1):144-162

109. Wolf MR, Eliseo-Arras RK, Brenner M, Nochajski TH (2016) "This will help your children": service providers' experiences with military families during cycles of deployment. J Fam Soc Work 20(1):1-15

110. Gray $H$ (2015) Militarism in the everyday: responses to domestic abuse in the British Armed Forces. Doctoral Dissertation, The London School of Economics and Political Science (LSE)

111. Rice VJ, Liu B (2017) Demographics, military status, and physical health as indicators of personal resilience among U.S. Active duty service members and veterans. Adv Intell Syst Comput. doi:10.1007/978-3-319-41652-6_40

112. Iversen A, Nikolaou V, Greenberg N, Unwin C, Hull L, Hotopf M, Dandeker C, Ross J, Wessely S (2005) What happens to British veterans when they leave the armed forces? Eur J Public Health 15(2):175-184

113. Herman A, Yarwood R (2014) From services to civilian: the geographies of veterans' post-military lives. Geoforum 53:41-50

114. Iversen A, Waterdrinker A, Fear N, Greenberg N, Barker C, Hotopf M, Hull L, Wessely S (2007) Factors associated with heavy alcohol consumption in the UK armed forces: data from a health survey of Gulf, Bosnia, and era veterans. Mil Med 172(9):956-961

115. Martin S (2014) Understanding military culture. BAMAATWORK. https://bamaatwork.wordpress.com/2014/08/29/under standing-military-culture/. Accessed 11 Jan 2017

116. Goodwin L, Wessely S, Hotopf M, Jones M, Greenberg N, Rona RJ, Hull L, Fear NT (2015) Are common mental disorders more prevalent in the UK serving military compared to the general working population? Psychol Med 45(9):1881-1891

117. Jonas S, Khalifeh H, Bebbington P, McManus S, Brugha T, Meltzer H, Howard L (2014) Gender differences in intimate partner violence and psychiatric disorders in England: results from the 2007 adult psychiatric morbidity survey. Epidemiol Psychiatr Sci 23(02):189-199

118. Hines DA (2007) Posttraumatic stress symptoms among men who sustain partner violence: an international multisite study of university students. Psychol Men Masc 8(4):225-239. doi:10. 1037/1524-9220.8.4.225

119. Randle AA, Graham CA (2011) A review of the evidence on the effects of intimate partner violence on men. Psychol Men Masc 12(2):97-111. doi:10.1037/a0021944

120. Archer J (2000) Sex differences in aggression between heterosexual partners: a meta-analytic review. Psychol Bull 126(5):651

121. Walby S, Towers J, Balderston S, Corradi C, Francis B, Heiskanen M, Helweg-Larsen K, Mergaert L, Olive P, Palmer E, Stöckl H, Strid S (2017) The concept and measurement of violence against women and men. Policy Press, Bristol, England

122. Cantos AL, Neidig PH, O'Leary KD (1994) Injuries of women and men in a treatment program for domestic violence. J Fam Violence 9(2):113-124

123. Langhinrichsen-Rohling J, Neidig P, Thorn G (1995) Violent marriages: gender differences in levels of current violence and past abuse. J Fam Violence 10(2):159-176

124. Barnish M (2004) Domestic violence: a literature review. HM Inspectorate of Probation, Manchester, England

125. World Health Organization (2013) Responding to intimate partner violence and sexual violence against women: WHO clinical and policy guidelines. World Health Organization, Geneva, Switzerland
126. Stöckl H, Devries K, Rotstein A, Abrahams N, Campbell J, Watts C, Moreno CG (2013) The global prevalence of intimate partner homicide: a systematic review. Lancet 382(9895):859-865

127. Tjaden PG, Thoennes N (2000) Extent, nature, and consequences of intimate partner violence: findings from the national violence against women survey, vol 181867. National Institute of Justice, Washington, DC

128. Walby S, Allen J, Simmons J (2004) Domestic violence, sexual assault and stalking: findings from the British Crime Survey. Home Office Research, Development and Statistics Directorate London, London

129. Straus M, Gelles R (1999) Physical violence in American families. Transaction, New Brunswick

130. Follingstad DR (2007) Rethinking current approaches to psychological abuse: conceptual and methodological issues. Aggress Violent Beh 12(4):439-458

131. Follingstad DR, Bush HM (2014) Measurement of intimate partner violence: a model for developing the gold standard. Psychol Violence 4(4):369

132. Maiuro R (2001) Sticks and stones may break my bones, but names will also hurt me: psychological abuse in domestically violent relationships. In: O'Leary KD, Maiuro RD (eds) Psychological abuse in violent domestic relations. Springer, New York, pp 9-20

133. Hamby SL (2005) Measuring gender differences in partner violence: implications from research on other forms of violent and socially undesirable behavior. Sex Roles 52(11-12):725-742

134. Maisto SA, McKay JR, Connors GJ (1990) Self-report issues in substance abuse: state of the art and future directions. Behav Assess 12(1):117-134

135. Hegarty K, Bush R, Sheehan M (2005) The composite abuse scale: further development and assessment of reliability and validity of a multidimensional partner abuse measure in clinical settings. Violence Vict 20(5):529-547

136. Ryan KM (2013) Issues of reliability in measuring intimate partner violence during courtship. Sex Roles 69(3-4):131-148

137. Follingstad DR, Edmundson M (2010) Is psychological abuse reciprocal in intimate relationships? Data from a national sample of American adults. J Fam Violence 25(5):495-508

138. Dobash RP, Dobash RE, Wilson M, Daly M (1992) The myth of sexual symmetry in marital violence. Soc Probl 39(1):71-91

139. Galdas PM, Cheater F, Marshall P (2005) Men and health helpseeking behaviour: literature review. J Adv Nurs 49(6):616-623

140. Ministry of Defence (2015) Armed forces domestic abuse: a handbook for civilian support services. https://www.gov.uk/ government/publications/armed-forces-domestic-abuse-a-hand book-for-civilian-support-services . Accessed 17 Nov 2016

141. Ministry of Defence (2015) JSP 770: Tri service operational and nonoperational welfare policy. https://www.gov.uk/government/ uploads/system/uploads/attachment_data/file/436113/20141215JSP_770_Final_v10-U.PDF. Accessed 24 Jan 2017

142. Violence NCoDaS (n.d.) Domestic violence intervention process model. Principle elements of strategic plan for most effectively addressing domestic violence matters within the Department of Defense. http://www.ncdsv.org/images/revstra tegicplan.pdf. Accessed 15 Oct 2016

143. Military OneSource (2017) The Family Advocacy Program. http://www.militaryonesource.mil/phases-military-leadership? content_id=266712. Accessed 20 July 2017

144. McCarroll JE, Castro S, Nelson EM, Fan Z, Evans PK, Rivera A (2008) Establishing and maintaining a volunteer victim advocate program to assist victims of domestic violence in the US Army. Mil Med 173(9):860-864 